Research Article

\title{
Early Onset Age Increased the Risk of Diabetic Retinopathy in Type 2 Diabetes Patients with Duration of 10-20 Years and HbA1C $\geq 7 \%$ : A Hospital-Based Case-Control Study
}

\author{
Jing Yuan $\mathbb{D}^{1}{ }^{1}$ Lin Zhang, ${ }^{1}$ Pu Jia, ${ }^{2}$ Zhong Xin, ${ }^{1}$ and Jin-Kui Yang $\mathbb{D}^{1}$ \\ ${ }^{1}$ Department of Endocrinology, Beijing Tongren Hospital, Capital Medical University, 1 Dong Jiao Min Xiang, \\ Beijing 100730, China \\ ${ }^{2}$ Department of Orthopaedics, Beijing Friendship Hospital, Capital Medical University, Beijing 100050, China
}

Correspondence should be addressed to Jin-Kui Yang; jkyang@ccmu.edu.cn

Received 22 January 2021; Accepted 1 June 2021; Published 11 June 2021

Academic Editor: Xiaojing Ma

Copyright (c) 2021 Jing Yuan et al. This is an open access article distributed under the Creative Commons Attribution License, which permits unrestricted use, distribution, and reproduction in any medium, provided the original work is properly cited.

Background. The relationship between onset age of diabetes and diabetic retinopathy (DR) is controversy and not concluded. Therefore, this hospital-based case-control study aimed to investigate the influence of diabetes onset age on the development of DR in patients with type 2 diabetes (T2D), independent of diabetic duration and HbA1c levels. Methods. A sample of 780 T2D patients with diabetic duration of 10-20 years and glycated hemoglobin (HbA1c) $\geq 7 \%$ were enrolled in the study. 338 T2D patients with onset age $\leq 45$ years were further selected as cases (early onset) and 79 with onset age $\geq 55$ years were chosen as controls (elderly onset). International Clinical Diabetic Retinopathy Disease Severity Scale was applied to estimate the severity of DR. Results. The prevalence of DR and proliferative diabetic retinopathy (PDR) was notably increased in the early onset group. When stratified by duration of diabetes, the impact of younger age on the risk of DR turned to be greatest in patients with diabetic duration $\geq 15$ years $(\mathrm{OR}=5.202,95 \% \mathrm{CI} 2.625-10.310)$. In groups stratified by HbA1c, the risk of DR was highest in patients with younger onset age and $\mathrm{HbAlc} \geq 9 \%(\mathrm{OR}=3.889,95 \% \mathrm{CI} 1.852-8.167)$. Compared with the elderly onset group, the risk of $\mathrm{DR}$ $(\mathrm{OR}=1.776,95 \% \mathrm{CI}=1.326-2.380, p<0.001)$ and $\mathrm{PDR}(\mathrm{OR}=1.605,95 \% \mathrm{CI}=1.106-2.329, p=0.013)$ in younger diagnosed patients was increased after multivariable adjustment. Conclusions. Age of onset was an independent risk factor for developing DR and PDR. This suggests that it is urgent to closely monitor and treat the metabolic disorders in younger T2D patients to delay the occurrence and progression of DR.

\section{Introduction}

Diabetic retinopathy (DR) is a prevalent and important microvascular complication of diabetes and widely considered as a major risk factor for vison loss and vison impairment in working-aged adults $[1,2]$, which could lead to decreased quality of life and increased financial burden of society. A Chinese epidemiological survey with 13473 diabetic patients demonstrated that the estimated prevalence of any DR and sight-threatening DR was $34.08 \%$ and $13.13 \%$, respectively [3]. Previous studies [4-7] have already offered insights that hyperglycemia and long duration of diabetes are well-known risk factors in the development and progression of DR. Other risk factors, such as hypertension [8] and high lipid levels [9], are also reported to be positively related with DR. Although accumulative studies have been conducted in order to better understand the pathogenic features of DR during the last decades, the risk factors of DR are still not equivocally elucidated.

There is a growing recognition that onset age of diabetes might be an underlying influencing factor to the risk of DR. Some studies reported that patients with early onset diabetes and with longer diabetic duration [10] or higher HbAlc [11] had an increased prevalence of DR. And another study indicated that type 2 diabetes (T2D) patients with onset age of 31-45 years, not $\leq 30$ years, had the highest susceptibility to DR [12]. Meanwhile, a study suggested that age at diabetes onset, diabetes could not predict the risk of DR at 17 years of 
diabetic duration [13]. Therefore, the issue is controversy and not concluded. To the best of our knowledge, a casecontrol study is not applied to date to minimize the influence of diabetic duration and glycemic control.

This study aimed to investigate whether onset age of diabetes was an independent risk factor to DR under a certain range of diabetic duration and HbA1c levels.

\section{Methods}

2.1. Study Population. This was a hospital-based case-control study. We screened a total of 1947 T2D patients aged $\geq 18$ years who were admitted to the Department of Endocrinology, Beijing Tongren Hospital, Capital Medical University, from January 2015 and May 2019, in Beijing, China. Based on inclusion criteria, a sample of 780 participants with diabetic duration of 10-20 years and glycated hemoglobin (HbA1c) $\geq 7 \%$ were selected in the study. And 129 subjects with no results of fundus photography were excluded from the study. After that, 338 T2D patients with onset age $\leq 45$ years were selected as cases (early onset) and 79 with onset age $\geq 55$ years were chosen as controls (elderly onset). The flowchart of the study is shown in Figure 1.

\subsection{Anthropometric and Laboratory Measurements.} Demographic details of patients were recorded, including age, sex, age of diagnosis, duration of diabetes, and smoking status. Smoking status was classified as never, former, or current. Antidiabetic treatment was also recorded. Body mass index (BMI) was calculated as the weight $(\mathrm{kg})$ divided by the square of height $\left(\mathrm{m}^{2}\right)$. Blood pressure (BP) was measured when the patients maintained a supine position and had a 10 minutes rest. Blood samples were taken at an overnight fasting status. Fasting blood glucose (FBG), serum creatinine (Scr), uric acid (UA), alanine aminotransferase (ALT), aspartate aminotransferase (AST), total cholesterol (TC), triglycerides (TG), and low-density lipoprotein (LDL) cholesterol concentrations were measured by an automated biochemical analyzer (Beckman company, US). HbA1c was assessed by high-performance liquid chromatography (VARIANT, BioRad Lab., Hercules, CA, US). Fasting blood C-peptide was measured by electrochemiluminescence (Roche, Germany).

2.3. Diabetic Retinopathy Assessment. As we described in the previous study, DR was determined using a TopconTRCNW7SF fundus camera (Topcon, Tokyo, Japan) ophthalmic digital imaging system ( 2 eyes $\times 7$ fields). These photographs were examined independently by two rigorously trained certified retinal photography graders while following quality assurance protocols. International Clinical Diabetic Retinopathy Disease Severity Scale [14] was applied to estimate the severity of DR as the following categories: nondiabetic retinopathy (NDR), nonproliferative diabetic retinopathy (NPDR), and proliferative diabetic retinopathy (PDR).
2.4. Statistical Analysis. Continuous variables were expressed as mean \pm standard deviation $(\bar{\chi} \pm s)$, and the comparison between 2 groups was made using a $t$-test. Categorical measures were expressed as percentage, and a chi-squared test was used for analysis. Binary logistic regression analysis was used to calculate crude and adjusted odds ratio (OR) to estimate the influencing factors of DR in hospitalized T2D patients. Statistical analyses were performed using SPSS ver. 17.0 software (SPSS Inc., Chicago, IL, US). A $p$ value $<0.05$ was considered to be a statistically significant difference.

\section{Results}

3.1. Characteristics of T2D Patients. The demographic and biochemical characteristics of the early onset and elderly onset group are given in Table 1. Compared with the elderly onset group, the prevalence of DR $(28.2 \%$ for controls vs. $54.7 \%$ for cases, $p<0.001)$ and PDR (14.1\% for controls vs. $25.5 \%$ for cases, $p<0.05$ ) was notably increased in the early onset group. The early onset group tended to have longer duration of diabetes, lower serum creatinine levels, less favorable lipid metabolism, and more current smokers $(p<0.001)$. More patients in the early onset group were treated by insulin $(p<0.001)$. There were no significant differences in sex, BMI, HbA1c, C-peptide, FBG, and UA $(p>0.05)$.

\subsection{Effects of Onset Age of Diabetes on Risk of DR Stratified by} Duration of Diabetes or $\mathrm{HbA1c}$. Patients were divided into two groups according to duration of diabetes $(<15$ years and $\geq 15$ years) and $\mathrm{HbA1c}(7-9 \%$ and $\geq 9 \%)$, respectively. As given in Table 2 and Figure 2, in groups stratified by duration of diabetes, the risk of DR in patients with younger age of diagnosis and diabetic duration $\geq 15$ years was greatest $(\mathrm{OR}=5.202$, 95\% CI 2.625-10.310), with approximate 2.7 times increase in the OR of retinopathy compared with patients of similar diabetic duration but diagnosed at $\geq 55$ years ( $O R=1.934,95 \%$ CI $0.666-5.619)$. Similarly, in groups stratified by HbAlc, the impact of early onset age on the risk of DR turned to be highest in T2D patients with $\mathrm{HbA1c} \geq 9 \%(\mathrm{OR}=3.889$, 95\% CI 1.852-8.167). The association between onset age and PDR stratified by diabetic duration or HbA1c was still significant but attenuated.

3.3. Association between Onset Age of Diabetes and the Risk of $D R$. The association between onset age of diabetes and the risk of DR was analyzed in 4 models (Table 3). Model 1 was a crude model. Model 2 was adjusted for sex and BMI. Model 3 was further adjusted for HbAlc and diabetic duration. Model 4 was additionally adjusted for serum creatinine, TG, and hypertension. Early onset age of diabetes was associated with higher risk of $\mathrm{DR}(\mathrm{OR}=1.749,95 \% \mathrm{CI}=1.338-2.287$, $p<0.001$ ) in univariate analysis (model 1). After adjustment for model 2, model 3, and model 4, the association remained statistical significant. Compared with the elderly onset group, the risk of PDR in younger diagnosed patients was also increased $(\mathrm{OR}=1.452, \quad 95 \% \quad \mathrm{CI}=1.003-2.0437$, $p=0.032$ ) for model 1 , and the age of onset was an independent risk factor for developing PDR after multivariable adjustment. 


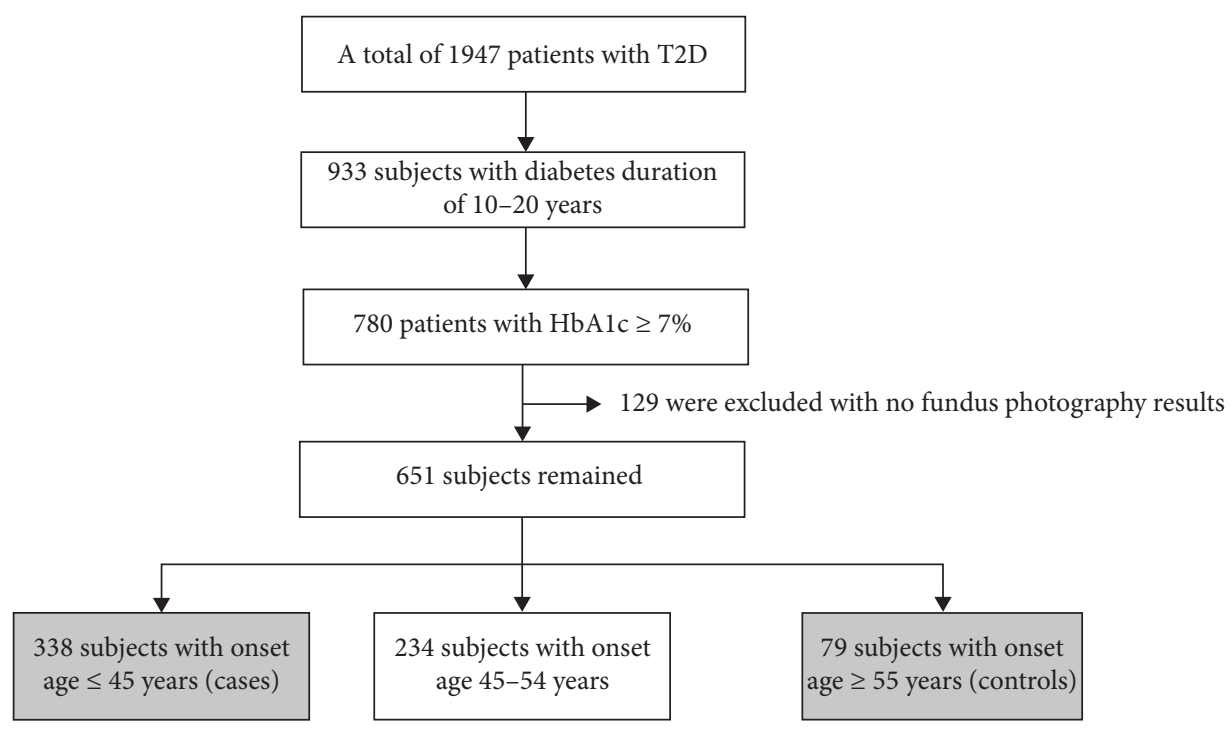

FIGURE 1: Flow chart of the study.

TABLE 1: Demographic and clinical characteristics of type 2 diabetes patients.

\begin{tabular}{|c|c|c|c|}
\hline & Onset age $\leq 45$ years $(n=338)$ & Onset age $\geq 55$ years $(n=79)$ & $p$ \\
\hline Age (years) & $53.58 \pm 6.65$ & $74.27 \pm 5.59$ & $<0.001$ \\
\hline Duration of diabetes (years) & $15.29 \pm 3.51$ & $13.66 \pm 3.57$ & $<0.001$ \\
\hline Male, $n(\%)$ & $210(62.1 \%)$ & $45(57.0 \%)$ & 0.396 \\
\hline \multicolumn{4}{|l|}{ Smoking status, $n(\%)$} \\
\hline Never & $178(52.7 \%)$ & $64(81.0 \%)$ & \multirow[t]{3}{*}{$<0.001$} \\
\hline Former & $44(13.0 \%)$ & $10(12.7 \%)$ & \\
\hline Current & $116(34.3 \%)$ & $5(6.3 \%)$ & \\
\hline BMI $\left(\mathrm{kg} / \mathrm{m}^{2}\right)$ & $25.87 \pm 3.34$ & $25.82 \pm 3.41$ & 0.908 \\
\hline HbAlc (\%) & $9.19 \pm 1.49$ & $9.05 \pm 1.58$ & 0.460 \\
\hline Fasting blood C-peptide $(\mathrm{ng} / \mathrm{ml})$ & $1.74 \pm 1.15$ & $2.07 \pm 1.31$ & 0.245 \\
\hline FBG $(\mathrm{mmol} / \mathrm{l})$ & $8.28 \pm 2.76$ & $8.37 \pm 2.46$ & 0.807 \\
\hline $\mathrm{SBP}(\mathrm{mmHg})$ & $133.78 \pm 17.14$ & $135.61 \pm 19.10$ & 0.485 \\
\hline DBP (mmHg) & $78.39 \pm 10.68$ & $74.93 \pm 12.39$ & 0.037 \\
\hline Scr $(\mathrm{mmol} / \mathrm{l})$ & $69.17 \pm 22.44$ & $80.15 \pm 26.27$ & $<0.001$ \\
\hline $\mathrm{UA}(\mathrm{mmol} / \mathrm{l})$ & $345.05 \pm 82.48$ & $333.87 \pm 90.75$ & 0.291 \\
\hline $\operatorname{ALT}(\mathrm{U} / \mathrm{l})$ & $22.82 \pm 15.56$ & $19.42 \pm 12.78$ & 0.162 \\
\hline AST (U/l) & $22.28 \pm 13.25$ & $21.44 \pm 9.64$ & 0.154 \\
\hline $\mathrm{TC}(\mathrm{mmol} / \mathrm{l})$ & $4.59 \pm 1.17$ & $3.96 \pm 0.93$ & $<0.001$ \\
\hline TG (mmol/l) & $2.21 \pm 1.83$ & $1.59 \pm 0.97$ & $<0.001$ \\
\hline $\mathrm{LDL}(\mathrm{mmol} / \mathrm{l})$ & $2.71 \pm 0.97$ & $2.29 \pm 0.79$ & $<0.001$ \\
\hline \multicolumn{4}{|l|}{ Antidiabetic treatment, $n(\%)$} \\
\hline Oral hypoglycemia agent & $318(94.1 \%)$ & $73(92.4 \%)$ & 0.579 \\
\hline Insulin & $304(89.9 \%)$ & $56(70.9 \%)$ & $<0.001$ \\
\hline GLP-1RA & $20(5.9 \%)$ & $2(2.5 \%)$ & 0.226 \\
\hline DR (\%) & $183(54.1 \%)$ & $22(27.8 \%)$ & $<0.001$ \\
\hline $\operatorname{PDR}(\%)$ & $86(25.4 \%)$ & $11(13.9 \%)$ & $<0.001$ \\
\hline
\end{tabular}

BMI, body mass index; HbA1c, glycated hemoglobin; FBG, fasting blood glucose; SBP, systolic blood pressure; DBP, diastolic blood pressure; Scr, serum creatinine; UA, uric acid; ALT, alanine aminotransferase; AST, aspartate aminotransferase; TC, total cholesterol; TG, triglycerides; LDL, low-density lipoprotein; GLP-1RA, GLP-1 receptor agonist; DR, diabetic retinopathy; PDR, proliferative diabetic retinopathy.

\section{Discussion}

In this hospital-based case-control study with T2D patients of diabetic duration $10-20$ years and $\mathrm{HbAlc} \geq 7 \%$, we demonstrated that the prevalence of DR and PDR was significantly higher in early onset T2D patients ( $\leq 45$ years) than in elderly onset T2D patients ( $\geq 55$ years). When further stratified by duration of diabetes and glycemic control, we found that the influence of early onset age on the risk of DR was highest in T2D patients with diabetic duration $\geq 15$ years (OR 5.202, 95\% CI 2.625-10.310) and HbAlc $\geq 9 \%$ (OR $3.889,95 \%$ CI 1.852-8.167). Age of onset was an independent risk factor for developing DR and PDR after multivariable adjustment with traditional risk factors of $\mathrm{DR}$. 
TABLE 2: Odds ratios of DR and PDR by onset age of T2D and category of diabetic duration or HbA1c.

\begin{tabular}{|c|c|c|c|c|}
\hline & \multicolumn{2}{|c|}{ DR } & \multicolumn{2}{|c|}{ PDR } \\
\hline & Early onset & Elderly onset & Early onset & Elderly onset \\
\hline \multicolumn{5}{|c|}{ Diabetes duration } \\
\hline$<15$ years & $2.819(1.446-5.495)$ & 1 (ref) & $3.039(1.140-8.102)$ & 1 (ref) \\
\hline$\geq 15$ years & $5.202(2.625-10.310)$ & $1.934(0.666-5.619)$ & $4.376(1.642-11.666)$ & $4.240(1.135-15.840)$ \\
\hline \multicolumn{5}{|l|}{ HbAlc (\%) } \\
\hline $7-9 \%$ & $1.914(0.910-4.026)$ & 1 (ref) & $1.797(0.702-4.598)$ & 1 (ref) \\
\hline$\geq 9 \%$ & $3.889(1.852-8.167)$ & $0.805(0.300-2.159)$ & $2.065(0.815-5.232)$ & $0.833(0.232-2.993)$ \\
\hline
\end{tabular}

DR, diabetic retinopathy; PDR, proliferative diabetic retinopathy; T2D, type 2 diabetes; HbA1c, glycated hemoglobin.

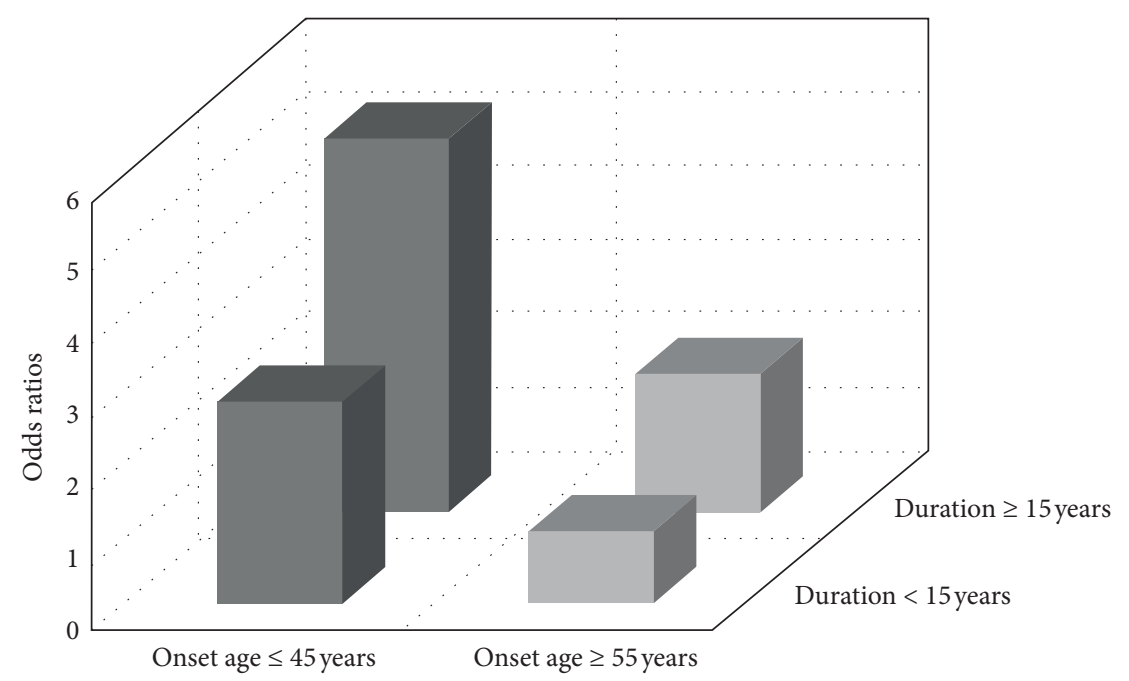

FIGURE 2: Odds ratios of DR by onset age of T2D and category of diabetic duration.

TABLE 3: The risk of DR and PDR according to onset age of T2D.

\begin{tabular}{lcccc}
\hline & DR & & PDR & \\
& OR (95\% CI) & $p$ & OR (95\% CI) & $p$ \\
\hline Model 1 & 1.749 & $<0.001$ & 1.452 & 0.032 \\
& $(1.338-2.287)$ & & $(1.033-2.043)$ & \\
Model 2 & 1.756 & $<0.001$ & $1.476(1.046-2.081)$ & 0.027 \\
& $(1.342-2.298)$ & & 1.403 & \\
Model 3 & 1.677 & $<0.001$ & $(0.990-1.986)$ & 0.057 \\
& $(1.273-2.207)$ & & & \\
Model 4 & 1.776 & $<0.001$ & $1.605(1.106-2.329)$ & 0.013 \\
& $(1.326-2.380)$ & & & \\
\hline
\end{tabular}

Model 1 was unadjusted. Model 2 was adjusted for sex and BMI. Model 3 was adjusted for sex, BMI, HbAlc, and diabetic duration. Model 4 was adjusted for sex, BMI, HbAlc, diabetic duration, serum creatinine, TG, and hypertension. DR, diabetic retinopathy; PDR, proliferative diabetic retinopathy; T2D, type 2 diabetes; OR, odds ratio; BMI, body mass index; HbA1c, glycated hemoglobin; TG, triglycerides.

Previous studies reported that early onset diabetes was more aggressive [15-17] and may be related to increased occurrence of diabetic microvascular [18]. Wong et al. [10] analyzed data from $624 \mathrm{~T} 2 \mathrm{D}$ patients with duration of $20-30$ years (group A) and 852 T2D patients with duration of 10-20 years (group B). The results suggested that early onset age ( $<45$ years) was an independent risk factor for DR after adjusted for traditional risk factors; however, disease duration, known as an important risk factor to DR, was not entered into the statistical model. Furthermore, the findings of the ADVANCE trial [19] reported a remarkable interaction between age or age at diagnosis and diabetes duration on the risk of microvascular events, and the highest risk of microvascular events was found in groups with the longest diabetes duration and the youngest age. In addition, a Chinese study with 29442 T2D patients of 77 tertiary hospitals indicated that the increased risk of DR in the early onset group was attributable to prolonged diabetes duration [20]. Therefore, it is essential to contain "diabetes duration" in the statistical model to evaluate the independent effect of early onset age on the development and progression of DR. In our study, we found that early onset age was associated with increased risk of DR, independent of diabetes duration, hyperglycemia, and other traditional risk factors of DR.

The relationship between age at onset and the risk of DR may not be limited to T2D. The FinnDiane Study [21] reported that compared to T1D patients with age at onset of 0-5 years and 15-40 years, group of 5-14 years had the highest risk of PDR, with $\mathrm{HbA1c}$, blood pressure, sex, and BMI as covariates. A retrospective cohort study [22] enrolled 153 T1D patients with onset of diabetes $<18$ years and suggested that older onset age was associated with development of DR 10 years after diagnosis. Some studies [23] indicated that puberty or delayed menarche could accelerate the progress of microvascular complications in T1D patients. Given the controversial results in T1D patients and 
underlying different mechanisms of DR between the two types of diabetes, it is inappropriate to mix T1D and T2D patients together for data analysis, as some previous studies conducted [24], which may not achieve accurate results. Therefore, the current study only enrolled T2D patients for analysis.

The potential mechanisms are still unclear. Several explanations may interpret the association between onset age and DR. First, more insulin could be secreted in $\beta$-cells of mature mice and humans than in young $\beta$-cells under the stimulation of high glucose concentrations [25-27], while some studies reported patients with better $\beta$-cell function had a reduced incidence of DR [28]. Second, the levels of angiogenin [29], vascular endothelial growth factor (VEGF) [30], and advanced glycation end products [31], which play a crucial role, in angiogenesis and in the development of DR, vary with age in diabetic patients. Third, the metabolic disorder may be more serious in early onset diabetes than in late-onset diabetes, which could lead to susceptibility to DR. Multiple factors such as genetic, socioeconomic, psychological, and behavioral factors [32] may involve in the pathogenesis.

There are several strengths and limitations of our study. One strength is that, to the best of our knowledge, it is the first case-control study to evaluate the association between onset age and DR. All patients selected in the study had long duration of diabetes (10-20 years) and high levels of blood glucose $(\mathrm{HbAlc}) \geq 7 \%$, which ensure the possibility to develop DR. Another strength is that the influence of diabetes onset age on the development of PDR was also investigated, which was previously conducted in only a few studies with T1D patients [21,33]. PDR is the most severe form of DR and could seriously affect the vision. We found that the prevalence of PDR was increased, and odds ratio of PDR was 1.605 (95\% CI: 1.106-2.329) in early onset diabetes. A limitation of the study was that as a hospitalized study, the T2D patients of our study may not be representative of the actual demographic profile of T2D patients in Beijing. Second, because of the nature of observation study, it was difficult to conceive the cause-effect relationship. Third, DR was measured only by fundus photography. Fundus fluorescence angiography and optical coherence tomography are needed in the future research.

\section{Conclusions}

In summary, our study shows a significant relationship between onset age and the risk of DR in T2D patients. This suggests that it is urgent to closely monitor and treat the metabolic disorders in younger T2D patients to delay the occurrence and progression of DR.

\section{Data Availability}

The data used to support the findings of this study are available from the corresponding author upon request.

\section{Ethical Approval}

The Ethical Committee of Beijing Tongren Hospital, Capital Medical University, approved the study.

\section{Consent}

Written informed consent was obtained from all study participants.

\section{Conflicts of Interest}

The authors declare that there are no conflicts of interest.

\section{Authors' Contributions}

Jin-Kui Yang designed the study and reviewed the manuscript. Jing Yuan collected the data. Jing Yuan, Pu Jia, and Zhong Xin analyzed and interpreted the data. Jing Yuan and Ling Zhang edited the text and contributed equally to this manuscript. All authors read and approved the final manuscript.

\section{Acknowledgments}

This work was supported by Beijing Municipal Administration of Hospitals Clinical Medicine Development of Special Funding Support (ZYLX201823) and Scientific Project of Beijing Municipal Science and Technology Commission (D171100002817005) to Jin-Kui Yang.

\section{References}

[1] B. E. Kobrin Klein, "Overview of epidemiologic studies of diabetic retinopathy," Ophthalmic Epidemiology, vol. 14, no. 4, pp. 179-183, 2007.

[2] J. W. Y. Yau, S. L. Rogers, R. Kawasaki et al., "Global prevalence and major risk factors of diabetic retinopathy," Diabetes Care, vol. 35, no. 3, pp. 556-564, 2012.

[3] Y. Liu, Y. Song, L. Tao et al., "Prevalence of diabetic retinopathy among 13473 patients with diabetes mellitus in China: a cross-sectional epidemiological survey in six provinces," BMJ Open, vol. 7, Article ID e013199, 2017.

[4] The Diabetes Control and Complications Trial Research Group, "The relationship of glycemic exposure (HbAlc) to the risk of development and progression of retinopathy in the diabetes control and complications trial," Diabetes, vol. 44, pp. 968-983, 1995.

[5] R. Klein, M. D. Knudtson, K. E. Lee, R. Gangnon, and B. E. K. Klein, "The Wisconsin epidemiologic study of diabetic retinopathy XXII," Ophthalmology, vol. 115, no. 11, pp. 1859-1868, 2008.

[6] U. Samuelsson, I. Steineck, and S. Gubbjornsdottir, "A high mean-HbAlc value 3-15 months after diagnosis of type 1 diabetes in childhood is related to metabolic control, macroalbuminuria, and retinopathy in early adulthood-a pilot study using two nation-wide population based quality registries," Pediatric Diabetes, vol. 15, no. 3, pp. 229-235, 2014.

[7] K. C. Donaghue, R. P. Wadwa, L. A. Dimeglio et al., "ISPAD Clinical Practice Consensus Guidelines 2014. Microvascular and macrovascular complications in children and adolescents," Pediatr Diabetes, vol. 15, no. 20, pp. 257-269, 2014. 
[8] J.-B. Zhou, Z.-H. Song, L. Bai, X.-R. Zhu, H.-B. Li, and J.-K. Yang, "Could intensive blood pressure control really reduce diabetic retinopathy outcomes? Evidence from metaanalysis and trial sequential analysis from randomized controlled trials," Diabetes Therapy, vol. 9, no. 5, pp. 2015-2027, 2018.

[9] Y.-R. Chung, S. W. Park, S.-Y. Choi et al., "Association of statin use and hypertriglyceridemia with diabetic macular edema in patients with type 2 diabetes and diabetic retinopathy," Cardiovascular Diabetology, vol. 16, no. 1, p. 4, 2017.

[10] J. Wong, L. Molyneaux, M. Constantino, S. M. Twigg, and D. K. Yue, "Timing is everything: age of onset influences longterm retinopathy risk in type 2 diabetes, independent of traditional risk factors," Diabetes Care, vol. 31, no. 10, pp. 1985-1990, 2008.

[11] R. O. Yeung, Y. Zhang, A. Luk et al., "Metabolic profiles and treatment gaps in young-onset type 2 diabetes in Asia (the JADE programme): a cross-sectional study of a prospective cohort," The Lancet Diabetes \& Endocrinology, vol. 2, no. 12, pp. 935-943, 2014.

[12] W. Zou, L. Ni, Q. Lu et al., "Diabetes onset at 31-45 years of age is associated with an increased risk of diabetic retinopathy in type 2 diabetes," Scientific Reports, vol. 6, no. 1, p. 38113, 2016.

[13] M. Tyrberg, L. Nyström, H. J. Arnqvist et al., "Overweight, hyperglycemia and tobacco use are modifiable risk factors for onset of retinopathy 9 and 17 years after the diagnosis of diabetes-a retrospective observational nation-wide cohort study," Diabetes Research and Clinical Practice, vol. 133, pp. 21-29, 2017.

[14] C. P. Wilkinson, F. L. Ferris, R. E. Klein et al., "Proposed international clinical diabetic retinopathy and diabetic macular edema disease severity scales," Ophthalmology, vol. 110, no. 9, pp. 1677-1682, 2003.

[15] O. Pinhas-Hamiel and P. Zeitler, "Acute and chronic complications of type 2 diabetes mellitus in children and adolescents,” The Lancet, vol. 369, no. 9575, pp. 1823-1831, 2007.

[16] T. A. Hillier and K. L. Pedula, "Complications in young adults with early-onset type 2 diabetes: losing the relative protection of youth," Diabetes Care, vol. 26, no. 11, pp. 2999-3005, 2003.

[17] M. Okudaira, H. Yokoyama, T. Otani, Y. Uchigata, and Y. Iwamoto, "Slightly elevated blood pressure as well as poor metabolic control are risk factors for the progression of retinopathy in early-onset Japanese Type 2 diabetes," Journal of Diabetes and Its Complications, vol. 14, no. 5, pp. 281-287, 2000.

[18] S. H. Song and C. A. Hardisty, "Early onset type 2 diabetes mellitus: a harbinger for complications in later years--clinical observation from a secondary care cohort," QJM, vol. 102, no. 11, pp. 799-806, 2009.

[19] S. Zoungas, M. Woodward, M. Woodward et al., "Impact of age, age at diagnosis and duration of diabetes on the risk of macrovascular and microvascular complications and death in type 2 diabetes," Diabetologia, vol. 57, no. 12, pp. 2465-2474, 2014.

[20] L. Li, L. Ji, X. Guo et al., "Prevalence of microvascular diseases among tertiary care Chinese with early versus late onset of type 2 diabetes," Journal of Diabetes and Its Complications, vol. 29, no. 1, pp. 32-37, 2015.

[21] K. Hietala, V. Harjutsalo, and C. Forsblom, "Age at onset and the risk of proliferative retinopathy in type 1 diabetes," $D i$ abetes Care, vol. 33, pp. 1315-1319, 2010.

[22] N.-K. Wang, C.-C. Lai, J.-P. Wang et al., "Risk factors associated with the development of retinopathy $10 \mathrm{yr}$ after the diagnosis of juvenile-onset type 1 diabetes in Taiwan: a cohort study from the CGJDES," Pediatric Diabetes, vol. 17, no. 6, pp. 407-416, 2016.

[23] V. Harjutsalo, C. Maric-Bilkan, C. Maric-Bilkan, C. Forsblom, and P.-H. Groop, "Age at menarche and the risk of diabetic microvascular complications in patients with type 1 diabetes," Diabetologia, vol. 59, no. 3, pp. 472-480, 2016.

[24] S. Zhang, J. Wang, C. Song, L. Zhu, and Y. Yu, "Lower prevalence of proliferative diabetic retinopathy in elderly onset patients with diabetes," Diabetes Research and Clinical Practice, vol. 125, pp. 47-52, 2017.

[25] A. Helman, D. Avrahami, A. Klochendler et al., "Effects of ageing and senescence on pancreatic $\beta$-cell function," Diabetes, Obesity and Metabolism, vol. 18, no. 1, pp. 58-62, 2016.

[26] D. Avrahami, C. Li, J. Zhang et al., "Aging-dependent demethylation of regulatory elements correlates with chromatin state and improved $\beta$ cell function," Cell Metabolism, vol. 22, no. 4, pp. 619-632, 2015.

[27] H. E. Arda, L. Li, J. Tsai et al., "Age-dependent pancreatic gene regulation reveals mechanisms governing human $\beta$ cell function," Cell Metabolism, vol. 23, no. 5, pp. 909-920, 2016.

[28] M. W. Steffes, S. Sibley, M. Jackson, and W. Thomas, "Betacell function and the development of diabetes-related complications in the diabetes control and complications trial," Diabetes Care, vol. 26, no. 3, pp. 832-836, 2003.

[29] F. Chiarelli, M. Pomilio, A. Mohn et al., "Serum angiogenin concentrations in young patients with diabetes mellitus," European Journal of Clinical Investigation, vol. 32, no. 2, pp. 110-114, 2002.

[30] F. Chiarelli, A. Spagnoli, F. Basciani et al., "Vascular endothelial growth factor (VEGF) in children, adolescents and young adults with Type 1 diabetes mellitus: relation to glycaemic control and microvascular complications," Diabetic Medicine, vol. 17, no. 9, pp. 650-656, 2000.

[31] F. Chiarelli, M. de Martino, A. Mezzetti et al., "Advanced glycation end products in children and adolescents with diabetes: relation to glycemic control and early microvascular complications," The Journal of Pediatrics, vol. 134, no. 4, pp. 486-491, 1999.

[32] Y. Zhang and G. Ning, "Young-onset type 2 diabetes mellitusa challenge for Asia," Nature Reviews Endocrinology, vol. 10, no. 12, pp. 703-704, 2014.

[33] M. Nordwall, M. Fredriksson, J. Ludvigsson, and H. J. Arnqvist, "Impact of age of onset, puberty, and glycemic control followed from diagnosis on incidence of retinopathy in type 1 diabetes: the VISS study," Diabetes Care, vol. 42, no. 4, pp. 609-616, 2019. 